\section{Pediatric Review - International Journal of Pediatric Research}

\title{
Hemophilia in children: a clinico-epidemiological profile and review
}

\author{
Singh M. ${ }^{1}$, Gupta L. ${ }^{2 *}$, Aneja G. ${ }^{3}$, Dayal R. ${ }^{4}$, Kumar N. ${ }^{5}$, Singh S. ${ }^{6}$, Nayak M. ${ }^{7}$, Kshitiz Sharma R. ${ }^{8}$ \\ DOI: https://doi.org/10.17511/ijpr.2020.i02.04

\begin{abstract}
${ }^{\mathbf{1}}$ Madhu Singh, Assistant Professor, ${ }^{2 *}$ L.K. Gupta, Associate Professor, Department of Pediatrics, Government Medical College, Firozabad, Uttar Pradesh, India. ${ }^{3}$ G.K. Aneja, D.M. Cardiology, Professor and Dean, ${ }^{4}$ R. Dayal, Professor and Head, ${ }^{5}$ Neeraj Kumar, Professor, ${ }^{6}$ Sheo Pratap Singh, Assistant Professor, ${ }^{\mathbf{7}}$ Madhu Nayak, Assistant Professor, ${ }^{\mathbf{8}}$ Ram Kshitiz Sharma, Assistant Professor; 1,3,4,5,6,7,8authors are
\end{abstract} \\ affiliated with the Department of Paediatrics, S.N. Medical College, Agra, Uttar Pradesh, India.
}

Introduction: Objective: To describe the clinical and epidemiological profile of hemophilia patients in the pediatric age group at a tertiary care center in Western Uttar Pradesh. Materials and Methods: This was an observational cross-sectional study that was conducted at the Department of Pediatrics, S.N. Medical College, Agra. Pediatric patients (0-18 years) with hemophilia admitted in the Pediatrics emergency ward for Anti- Hemophilic factor transfusion or having a history of such type of transfusion during the year 2018-19 were consecutively enrolled in the present study. A detailed clinical history was taken from the parent or guardian accompanying the case. Results: Out of the total 93 pediatric hemophilia patients, the majority 61\% (57/93) belonged to 11 to 18 years of age group. $2.1 \%$ (2/93) were< 1 year of age. $17.2 \%(16 / 93)$ were in 1-5 years of age group. $19.35 \%$ were in $6-10$ years of age group. Out of the total, $98.9 \%$ (92/93) were males while only $1.07 \%$ (1/93) were female. Type A hemophilia was observed in $87.09 \%(81 / 93)$ patients while Type B hemophilia was present in only $12.9 \%$ (12/93) patients. Out of 81 Type-A hemophilia patients, only one had developed Inhibitor (1.07\%). None among Type B hemophilia patients had Inhibitors. Out of total cases, only one patient (1.07\%) had von Willebrand Deficiency.91\%(85/93) patients presented with joint bleed. The knee joint was the most commonly affected joint, as observed in $64.7 \%$ (55/85) patients. Conclusion: A better insight into the disease prevalence is pivotal for early diagnosis and to provide quality care to all hemophilia patients. The development of an inhibitor is a major concern. Physiotherapy and RICE(Rest, Ice, Compression and elevation) should be emphasized for the better quality of life and preventing joint hemarthrosis.

Keywords: Clinico- epidemiological profile, Hemophilia, Inhibitors, Rest, Ice, Compression and Elevation (RICE)

Corresponding Author

L.K. Gupta, Associate Professor, Department of Pediatrics, Government Medical College, Firozabad, Uttar Pradesh, India.

Email: guptaneeta98@gmail.com
How to Cite this Article

To Browse

Singh M, Gupta LK, Aneja GK, Dayal R, Kumar N, Singh SP, Nayak M, Sharma RK. Hemophilia in children: a clinico-epidemiological profile and review. Pediatric Rev Int J Pediatr Res. 2020;7(2):66-72. Available From https://pediatrics.medresearch.in/index.php/ijpr/arti cle/view/575

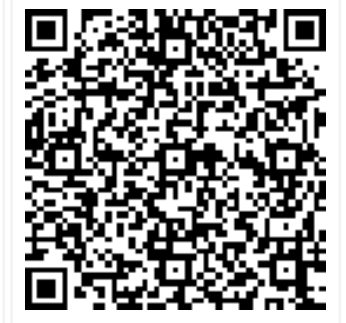

Manuscript Received 2020-01-25

Conflict of Interest No
Review Round 1 2020-02-08

Funding $\mathrm{Nil}$
Review Round 2 2020-02-13

Ethical Approval Yes
Review Round 3

Accepted 2020-02-17

Plagiarism X-checker $4 \%$ (c) 2020 by Madhu Singh, L.K. Gupta, G.K. Aneja, R. Dayal, Neeraj Kumar, Sheo Pratap Singh, Madhu Nayak, Ram Kshitiz
Sharma and Published by Siddharth Health Research and Social Welfare Society. This is an Open Access article licensed under a Creative Commons Attribution 4.0 International License https://creativecommons.org/licenses/by/4.0/ unported [CC BY 4.0]. 


\section{Introduction}

Hemophilia is an X-linked recessive bleeding disorder caused by the deficiency of coagulation factors VIII, IX, or XI. Hemophilia A (Factor VIII deficiency) and Hemophilia B (Factor IX deficiency) are the commonest and the serious ones and their clinical presentation mostly overlap. Factor XI deficiency is commonly known as Hemophilia C. It is the milder variant and mostly seen in Ashkenazi Jews.

Deficiency of Factor VIII or Factor IX leads to a defective intrinsic coagulation pathway (decreased and delayed generation of thrombin, defective clot formation, and hemorrhagic diathesis). Being an Xlinked recessive disorder, males are affected and females are asymptomatic or mildly affected carriers.

Epidemiology: In 2018, there was a 204\% increase in the number of people with bleeding disorders identified since 1999 globally [Figure 1]. World Federation of Hemophilia (WFH) report on the annual global survey 2018, which included 125 national member organizations (NMOs), identified nearly 210,454 hemophilia patients. Out of the global total, nearly, 173,711(82.54\%) had hemophilia A while 34,289 (16\%) had hemophilia B. 78,547 were identified to have VWD. The total number of people with hemophilia A with clinically identified inhibitors was 6,929. A number of people with hemophilia B with clinically identified inhibitors was only 394 [1].

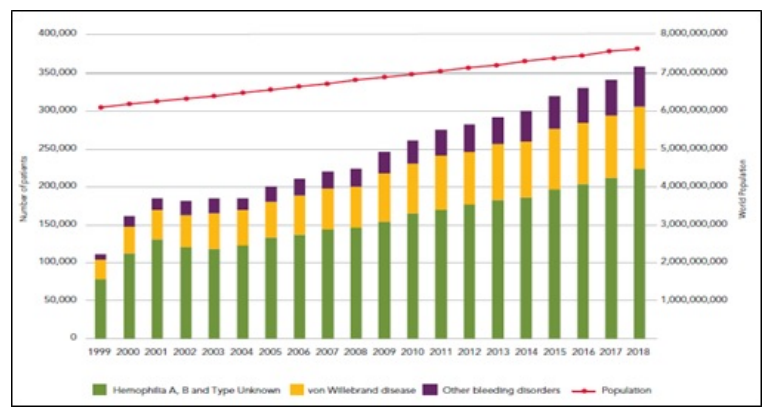

Figure-1: Identified patients over time - all bleeding disorders

Source: Report on the Annual Global Survey 2018 is published by the World Federation of Hemophilia.

In the year 2018, nearly 20,778 people were suffering from Hemophilia in India, out of the which, 17,606(84.73\%) had Hemophilia A while 2,715(13.06\%) had Hemophilia B.
6676 people had von Willebrand disease. The agewise distribution of Hemophilia A in age groups 0-4, 5-13 and $14-18$ was $2 \%, 14 \%$, and $10 \%$ respectively, while for Hemophilia B in age groups $0-4,5-13$ and $14-18$ was $2 \%, 13 \%$ and $12 \%$ respectively [1].

Hemophilia should be clinically suspected in any child with joint bleeds, easy bruising, unprovoked deep-seated bleeds or prolonged/ excess bleeds following surgery or trauma. Intracranial hemorrhage in neonate might be the earliest and devastating manifestation which is seen in only 2 $4 \%$ of cases [2]. The neonate may present with subgaleal bleeds and cephalhematoma.

These early presentations may be associated with traumatic deliveries (like forceps or vacuum extraction), and underlying hemophilia should not be missed as a part of initial diagnosis [2]. Prolonged bleeding after circumcision or a muscle hematoma following vaccination in an infant should also raise suspicion of hemophilia. Only about onethird of cases of hemophilia are due to spontaneous mutations without a prior family history.

In the most ideal situation, the remaining two-thirds should be diagnosed antenatally or at birth because of family history. However, in the Indian setting, the first clinical presentation is often a post-traumatic bleed, usually, hemarthrosis or skin bleed [3].

\section{Material and Methods}

Type of Study: This retrospective record-based cross-sectional study was conducted in S.N.Medical College, Agra. The data of all confirmed cases of hemophilia in the pediatric age group ( $0-18$ years) were examined by the investigators.

Duration of Study: Pediatric patients (0-18 years) with hemophilia admitted in Pediatrics emergency ward for Anti- Hemophilic factor transfusion or having a history of such type of transfusion during the year 2018-19 were consecutively enrolled in the current study.

Sampling Methods And Sample Size: The information regarding sociodemographic details of the patients, type of hemophilia, risk factors such as the family history of genetic disorders, history of consanguinity among parents, symptoms, signs, and complications associated with hemophilia, hematological reports, and management practices were recorded in a predesigned validated proforma. 
Data Collection Procedure: This retrospective study involved patients who were admitted to the Pediatric emergency Ward, Department of Pediatrics, S.N. Medical College, Agra for the purpose of factor transfusion. Ethical clearance was taken from an ethical society. Parents or guardians were informed about the purpose of the study. For each patient, a detailed history was taken from the mother or the attendant. After taking a brief history preliminary selection was done, and the purpose of the study was explained in detail to its subject. After taking consent from the parents, data was collected, which included sex, age at presentation, age at diagnosis and clinical symptoms at presentation. A thorough physical examination was done in each patient.

Ethical Consideration and Permission: The study was conducted in the Department of Pediatrics, S.N. Medical College, Agra after obtaining ethical clearance from the Ethical Committee of S.N. Medical College, Agra.

\section{Results}

Out of the total 93 pediatric hemophilia patients, the majority $61 \%(57 / 93)$ belonged to 11 to 18 years of age group. $2.1 \%$ (2/93) were $<1$ year of age. $17.2 \%(16 / 93)$ were in $1-5$ years of age group. $19.35 \%$ were in $6-10$ years of age group (Table-1).

Table-1: Epidemiological profile of hemophilia patients.

\begin{tabular}{|c|c|c|}
\hline & & N (\%) \\
\hline \multirow[t]{4}{*}{ Age group(yrs) } & $<1$ & $2(2.1)$ \\
\hline & $1-5$ & $16(17.2)$ \\
\hline & $6-10$ & $18(19.35)$ \\
\hline & $11-18$ & $57(61)$ \\
\hline \multirow[t]{2}{*}{ Gender } & Male & $92(98.92)$ \\
\hline & Female & $1(1.07)$ \\
\hline \multirow[t]{2}{*}{ Socioeconomic status } & Above the poverty line & $52(55.91)$ \\
\hline & Below poverty line & $41(44.08)$ \\
\hline \multirow[t]{2}{*}{ Residence } & Urban & $63(67.74)$ \\
\hline & Rural & $30(32.25)$ \\
\hline
\end{tabular}

Out of a total of $98.92 \%$ (92/93) were males while only $1.07 \%(1 / 93)$ were female (Table-1). 52 children out of 93 were from above the poverty line while $41 / 93$ were from low socioeconomic status. $63 / 93$ belonged to the urban area while 30 came from rural areas in the current study population (Table-1). Type A hemophilia was observed in 87.09\% (81/93) patients while Type B hemophilia was present in only $12.9 \%(12 / 93)$ patients (Table
-2). Out of 81 Type A hemophilia patients, only one had developed Inhibitor (1.07\%). None among Type $B$ hemophilia patient had Inhibitors (Table-2). Out of total cases, only one patient $(0.01 \%)$ had von Willebrand Deficiency (Table-2).

Table-2: Types of hemophilia in the study population.

\begin{tabular}{|l|l|}
\hline \multicolumn{1}{|c|}{ Type of hemophilia } & \multicolumn{1}{c|}{ N (\%) } \\
\hline Hemophilia A & $81(87.09)$ \\
\hline Hemophilia B & $12(12.09)$ \\
\hline Hemophilia A + inhibitor & $1(1.07)$ \\
\hline Hemophilia B + inhibitor & $0(0.00)$ \\
\hline vWF deficiency & $1(1.07)$ \\
\hline
\end{tabular}

$91.39 \%(85 / 93)$ patients presented with joint bleed. 20.4 (19\%) patients had hematuria and $3.22 \%$ (3/93) patients presented with bleeding from scalp. 1 patient each presented with a bleed from tongue, finger, pelvis, and gum (Table-3).

The knee joint was the most commonly affected joint as observed in $64.7 \%(55 / 85)$ patients, followed by the elbow (15.29), ankle (11.76\%), shoulder(5.88\%) and wrist joint(2.35\%) (Table-4).

Table-3: Clinical profile of hemophilia patients in the study population.

\begin{tabular}{|l|l|}
\hline \multicolumn{1}{|c|}{ Clinical features } & \multicolumn{1}{c|}{ N (\%) } \\
\hline Joint bleed & $85(91.39)$ \\
\hline Hematuria & $19(20.4)$ \\
\hline Scalp bleed & $3(3.22)$ \\
\hline Tongue bleed & $1(1.07)$ \\
\hline Finger bleed & $1(1.07)$ \\
\hline Pelvis bleed & $1(1.07)$ \\
\hline Gum bleed & $1(1.07)$ \\
\hline
\end{tabular}

Table-4: Join the involvement of hemophilia patients in the study population.

\begin{tabular}{|l|l|}
\hline & \\
\hline Knee & $55(64.7)$ \\
\hline Elbow & $13(15.29)$ \\
\hline Ankle & $10(11.76)$ \\
\hline Shoulder & $5(5.88)$ \\
\hline Wrist & $2(2.35)$ \\
\hline
\end{tabular}

\section{Discussion}

The hallmark of hemophilia is deep bleeding into joints and muscles, though bleeding can occur anywhere. In infants and toddlers, the ankle is the most common site followed by knees. In older children, knees and elbows are frequently involved. The most common joint involved in patients not taking prophylaxis is the knee, followed by elbow, 
Ankle, shoulder, and wrist. In those on prophylaxis, the ankle is commonly involved. Occasionally, mucosal bleeds (epistaxis, gums, gastrointestinal, genitourinary) might be the presenting features. Central nervous system (CNS) bleed may occur at any age, insidiously or following mild trauma. A suspected intracranial bleed in a diagnosed hemophilia patient must be first treated with factor concentrates even before confirmation by neuroimaging.

The bleeding phenotype differs based on the factor level and accordingly, hemophilia is classified into mild $(>5 \%)$, moderate $(1-5 \%)$ and severe $(<1 \%)$ [4]. Severe hemophilia presents with spontaneous bleeds, especially from CNS, muscle, joints, etc. Nearly $50 \%$ of patients with severe hemophilia manifest by 6-8 months of age (with an increase in physical activity). Moderate hemophilia has heterogeneous manifestations. People with mild hemophilia usually bleed only after major surgery or trauma. Female carriers of hemophilia too can present with bleeding in the form of menorrhagia, skin bruising and postsurgical or peripartum hemorrhage [5].

Initial screening blood investigations (with reference ranges) for any child with the suspected bleeding disorder include platelet count $(150-450 \times 103 / \mu \mathrm{L})$; prothrombin time (PT) (11-15 seconds), and activated partial thromboplastin time (aPTT) (29-35 seconds). In hemophilia, screening tests reveal a prolonged aPTT with a normal PT and platelet count [6]. The other inherited conditions with similar screening results include von Willebrand disease (certain types), factors XI, XII, high molecular weight kininogen and prekallikrein deficiencies.

Mixing study is a simple test performed by mixing plasma from the patient with pooled normal plasma in $1: 1$ proportion and repeating aPTT after incubating for 30-60 minutes. Correction of aPTT after mixing suggests a deficiency in any of the factors in the intrinsic pathway. Prolonged aPTT may not get corrected with mixing study in case of the presence of antibodies to factor VIII or IX, lupus anticoagulant or heparinized sample [6]. Specific factor assays for factor VIII or IX should be done for specific diagnosis and ascertaining its severity. Allfemale carriers in the family should get their factor level tested as they can have levels ranging from normal to borderline (40-60\%). Those with borderline levels can have increased bleeding tendency, especially during pregnancy, invasive procedures and trauma [5].
They might also require factor replacement during such conditions. Menorrhagia is frequently noted in such carriers, which may respond to anti-fibrinolytic or oral contraceptives. Rarely, females can have hemophilia (factor level $<40 \%$ ). The Mini Report 1 of Annual Global Survey published by WFH in April 2017 has identified nearly 4000 females with Hemophilia A and 1300 females with Hemophilia B [7]. The most common bleeding disorder for women and girls is VWD[8]. During the 2007 to 2015 period, there was a $79 \%$ increase in the number of women identified with VWD $[9,10]$.

Any strenuous exercise or intake of drugs like aspirin prior to sample collection should be preferably avoided. Ideally, the samples should be stored at 20-25oC and processed within 4 hours of collection, as delayed processing gives erroneous results [11]. If the suspected transport time to the laboratory is $>4$ hours, the sample is centrifuged immediately to separate platelet-poor plasma and transport frozen plasma in dry ice. Specimens can be stored at $-20^{\circ} \mathrm{C}$ for up to 2 weeks or at $-70^{\circ} \mathrm{C}$ for up to 6 months. Contamination with heparin should be avoided. The sample should be avoided by central catheters. Blood: citrate ratio of $9: 1$ should be maintained while collecting the blood sample in a citrate vial.

Since the normal factor IX level is low in the neonatal period, diagnosis of hemophilia $B$ in the neonatal period and infancy may be challenging. Age-specific cut-off levels should, therefore, be used. While a diagnosis of moderate and severe cases is still possible, a suspected mild hemophilia $B$ requires confirmation at 3-6 months of age.

Management of hemophilia A or B requires a lifelong replacement of coagulation proteins in the form of Factor VIII and Factor IX respectively. The most feared complication of this therapy is the development of inhibitors that neutralize the clotting factors[12]. The risk of developing inhibitors is greatest in previously untreated patients with severe hemophilia $A$; however, hemophilia $B$ patients can also develop inhibitors [13]. Inhibitor development is multifactorial. Most patients are expected to develop inhibitors within the first 10-15 exposure days, reaching a plateau after 50-75 exposure days[14]. Genetic factors have been associated with a higher risk of inhibitor development. Patients with large deletions, nonsense mutations, and intron 22 inversions have a seven to tenfold higher risk of inhibitor development [15]. 
Young age and the reason for the first infusion along with the intensity of treatment could be important risk factors for the development of inhibitors.

Factor replacement is the mainstay of treatment for hemophilia. Minor bleeds in a joint, soft tissue or muscle can be slowed by using First Aid. The steps of this First Aid to slow bleeding are called RICE which stands for Rest, Ice, Compression, and Elevation. It is a common treatment used for many types of injuries, including those suffered by professional athletes.

Rest: While the joint is bleeding, it should be rested. In the event of an ankle or knee bleed, they should keep off their feet as much as possible and not do any physical activities. They should keep a set of crutches at home to use when a bleed starts. In hemophilia care, the " $\mathrm{R}$ " in RICE can also mean "Replacement of clotting factor".

Ice: To help control swelling and reduce the pain they should apply ice. They can use crushed ice in a plastic bag or a bag of frozen vegetables, wrapped in a towel. It is very important not to apply the ice for too long. Ten or fifteen minutes at a time, every 2 hours, is long enough. The "I" in RICE can also mean "Immobilization". The joint is held still (immobilized). When there is a lot of swelling, and if the movement is difficult and painful, the joint should be immobilized by a half-cast or a splint for short periods only, about 2 or 3 days. If the joint is kept immobilized for longer periods, the joint can become stiff and the muscles can become weak, making the joint more prone to re-injury.

Compression: They should apply pressure on the area that is bleeding to help 'pinch off' the blood vessels and slow down the bleeding. Compression of a joint or muscle bleed is done by using an elastic bandage (tensor). They should wrap the injured part in a figure-eight pattern. It is important that they watch carefully for coolness, numbness or a change in color in fingers or toes. If any of these symptoms occur, they should remove the bandage and re-wrap it less tightly. An alternative is a Tubigrip $®$ sleeve. If the injury is very sore, they may find compression increases the pain. If this happens, they should rewrap the injury less tightly, or remove the bandage altogether.

Elevation: Placing the injured limb at a level higher than the heart helps decrease the pressure in the blood vessels and helps to slow the bleeding. They can rest their injured arm or leg on pillows.
Roche's Emicizumab, a monoclonal antibody is now available in India. Emicizumab prophylaxis is found to reduce the number of bleeding episodes in people with Hemophilia A and inhibitors of factor VIII.

In the current study, $87.09 \%$ of children, out of the total were found to have Hemophilia $A$ and only $12.9 \%$ were having Hemophilia B. Mishra et al had similar results. They observed that hemophilia $A$ $(88.3 \%)$ was the most common type of hemophilia as compared to hemophilia B [16]. Agarwal et al also found the percentage of hemophilia $A$ is $78.6 \%$ (236/ 300 cases) further confirming our data [17]. Kulkarni et al state that the majority (81\%) of their hemophilia patients belonged to Hemophilia $A$ and only $19 \%$ were having hemophilia B [18].

In the current study, $91.39 \%(85 / 93)$ patients presented with joint bleed. 20.4 (19\%) patients had hematuria and $3.22 \%(3 / 93)$ patients presented with bleeding from scalp. 1 patient each presented with a bleed from tongue, finger, pelvis, and gum. In an Indian study by Kar et al, in 148 patients with severe hemophilia $A$, the knee joint was affected in all[19]. The long-term consequences of repeated joint bleeds are the development of progressive joint damage and disability.

In the current study, 1 (1.07\%) out of 93 children was found to have an inhibitor. A systematic review reported the prevalence of patients with inhibitors between 5 to 7 percent with the risk of inhibitor development being higher in patients with severe rather than mild or moderate hemophilia $A$. The only constraint in the current study is the small sample size. Further studies are needed to detect inhibitors in all patients.

\section{Conclusion}

Knowledge of disease prevalence in a particular region is vital for establishing an early diagnosis. Lack of antenatal screening and lack of resources is the biggest hindrance in diagnosing a bleeding disorder in developing countries like India. A high index of suspicion should thus be kept while detecting hemophilia.

Inhibitor screening is also a must in the present scenario because delayed detection can lead to wastage of factors and resources. Physiotherapy and RICE (Rest, Ice, Compression and elevation) are two important aspects of care that should be emphasized in each hemophilia patient for the better quality of life and preventing joint hemarthrosis. 


\section{What does this study add to the existing knowledge?}

The current study is conducted to evaluate the clinical-epidemiological profile of Hemophilia patients in the pediatric age group.

Such studies are pivotal to establish an early diagnosis of patients as well as to prevent complications such as joint swelling, deformity and fatal bleeding in registered patients by active and timely intervention.

Nonresponse to treatment likely suggests the development of inhibitors and thus these researches are a must-have insight into basic pathology and subsequent treatment with available resources.

\section{Author's contributions}

Dr. Madhu Singh: Concept, study design, Dr. L.K. Gupta: Study design, Dr. G.K. Aneja: Data compilation, Prof (Dr). R. Dayal: Statistical analysis, Prof (Dr). Neeraj Kumar: Statistical analysis, Dr. Sheo Pratap Singh: Manuscript review, Dr. Madhu Nayak: Data analysis, Dr. Ram Kshitiz Sharma: Manuscript preparation

\section{Acknowledgments}

The authors sincerely thank Professor Dr. G.K Aneja, (Dean, S.N. Medical College, Agra) and Professor Dr. R. Dayal (Head, Department of Pediatrics, S.N.MedicalCollege, Agra) for continuous encouragement and inspiration to complete the work. The authors especially would like to thank Prof (Dr). Neeraj Kumar, and Dr. L.K. Gupta who have guided the work. Authors would also like to thank their colleagues for valuable suggestions and encouragement throughout the research work.

\section{Reference}

01. World Federation of Hemophilia. Report on the Annual Global Survey 2018. Montreal, CanadaWFH. 2019. Available at [Article][Crossref][PubMed] [Google Scholar]

02. Davies J, Kadir RA. Mode of delivery and cranial bleeding in newborns with haemophilia- A systematic review and meta-analysis of the literature. Haemophilia. 2016;22(1)32-38. doi: 10.1111/hae.12726 [Crossref][PubMed][Google Scholar]
03. Payal V, Sharma P, Goyal V, Jora R, Parakh M, Payal D. Clinical profile of hemophilia patients in Jodhpur region. Asian J Transfus Sci. 2016;10(1)101-104. Available from: [Article] [Crossref][PubMed][Google Scholar]

04. Witmer C, Young G. Factor VIII inhibitors in hemophilia A- rationale and latest evidence. Ther Adv Hematol. 2013;4(1)59-72. doi: 10.1177/20406 20712464509 [Crossref][PubMed][Google Scholar]

05. Paroskie A, Gailani D, DeBaun MR, Sidonio RF Jr. A cross-sectional study of bleeding phenotype in haemophilia A carriers. Br J Haematol. 2015;170(2) 223-8. doi: 10.1111/bjh.13423 [Crossref][PubMed] [Google Scholar]

06. Kamal AH, Tefferi A, Pruthi RK. How to interpret and pursue an abnormal prothrombin time, activated partial thromboplastin time, and bleeding time in adults. Mayo Clin Proc. 2007;82(7)864-873. doi: 10.4065/82.7.864 [Crossref][PubMed][Google Scholar]

07. World Federation of Hemophilia. Report on the Annual Global Survey 2017. Montreal, CanadaWFH. 2018. Available at [Article][Crossref][PubMed] [Google Scholar]

08. World Federation of Hemophilia. Annual Global Survey, Mini Report 1. WHF. April 2017. Available at [Article][Crossref][PubMed][Google Scholar]

09. James AH, Kouides PA, Abdul-Kadir R, Edlund M, Federici $A B$, Halimeh $S$, et al. Von Willebrand disease and other bleeding disorders in womenconsensus on diagnosis and management from an international expert panel. Am J Obstet Gynecol. 2009;201(1)12-e1. [Crossref][PubMed][Google Scholar]

10. World Federation of Hemophilia. Report on the Annual Global Survey 2015. Montreal, CanadaWFH. 2016. Available at [Article][Crossref][PubMed] [Google Scholar]

11. Srivastava A, Brewer AK, Mauser-Bunschoten EP, Key NS, Kitchen S, Llinas A, et al. Guidelines for the Management of Hemophilia. Haemophilia. 2013;19(1)1-4. doi: 10.1111/j.1365-2516.2012. 02909.x [Crossref][PubMed][Google Scholar]

12. Ananyeva NM, Lee TK, Jain N, Shima M, Saenko EL. Inhibitors in hemophilia $A^{-}$advances in elucidation of inhibitory mechanisms and in inhibitor management with bypassing agents. Semin Thromb Haemost. 2009;35(8)735-751. doi: 10.1055/s0029-1245106 [Crossref][PubMed7「Gooqle Scholar] 
13. Santagostino E, Morfini M, Auerswald GK-H, et al. Paediatric haemophilia with inhibitors- existing management options, treatment gaps and unmet needs. Haemophilia. 2009;15(5)983-989. [Crossref] [PubMed][Google Scholar]

14. Wight J, Paisley S. The epidemiology of inhibitors in haemophilia A- a systematic review. Haemophilia. 2003;9(4)418-435. doi: 10.1046/j.1365-2516.2003.00780.x [Crossref] [PubMed][Google Scholar]

15. Gouw SC, van den Berg HM. The multifactorial etiology of inhibitor development in hemophiliagenetics and environment. Semin Thromb Haemost. 2009;35(8)723-734. doi: 10.1055/s-0029-1245105 [Crossref][PubMed][Google Scholar]

16. Mishra S, Kumar S, Panwar A, Bhagchandani D, Aneja GK, Verma N, et al. A clinical profile of hemophilia patients and assessment of their quality of life in Western Uttar Pradesh, India- an observational study. Med J DY Patil Univ. 2016;9(3)320-324. doi: 10.4103/09752870.182501 [Crossref][PubMed][Google Scholar]
17. Agarwal MB, Mehta BC, Bhanotra PC. Classical hemophilia a study of 236 cases from 212 unrelated families. J Assoc Physicians India. 1981;29(5)385389. [Crossref][PubMed][Google Scholar]

18. Kulkarni R, Soucie JM, Lusher J, Presley R, Shapiro A, Gill J, et al. Sites of initial bleeding episodes, mode of delivery and age of diagnosis in babies with haemophilia diagnosed before the age of 2 years- a report from the centers for disease control and prevention's (CDC) universal data collection (UDC) project. Haemophilia. 2009;15(6)1281-1290. doi: 10.1111/j.13652516.2009.02074.x [Crossref][PubMed][Google Scholar]

19. Kar A, Mirkazemi R, Singh $P$, Potnis-Lele $M$, Lohade $S$, Lalwani $A$, et al. Disability in Indian patients with haemophilia. Haemophilia. 2007;13(4)398-404. doi: 10.1111/j.13652516.2007.01483.x [Crossref][PubMed][Google Scholar]

20. Wight J, Paisley S. The epidemiology of inhibitors in haemophilia A- a systematic review. Haemophilia. 2003;9(4)418-435. doi: 10.1046/j.1365-2516.2003.00780.x [Crossref] [PubMed][Google Scholar] 